\title{
Combined hepatocellular carcinoma and cholangiocarcinoma (biphenotypic) tumors: clinical characteristics, imaging features of contrast-enhanced ultrasound and computed tomography
}

Rui Li ${ }^{1 *}$, Dan Yang ${ }^{2}$, Chun-Lin Tang ${ }^{2}$, Ping Cai ${ }^{3}$, Kuan-sheng Ma ${ }^{1}$, Shi-Yi Ding ${ }^{3}$, Xiao-Hang Zhang ${ }^{2}$, De-Yu Guo ${ }^{4}$ and Xiao-Chu Yan ${ }^{4}$

\begin{abstract}
Background: Combined hepatocellular-cholangiocarcinoma (CHCC-CC) is an uncommon primary liver malignancy and little known about the clinical and imaging characteristics of CHCC-CC. We aim to define the demographics, imaging features of CHCC-CC on contrast-enhanced ultrasound (CEUS) and contrast-enhanced computed tomography $(\mathrm{CT})$ in this study.

Methods: From January 2005 to December 2014, 45 patients with pathologically proven cHCC-CC who underwent preoperative CEUS and 43 patients who had additional CT scan in our institution were included. A retrospective review of the imaging studies and clinical data in these patients was conducted.
\end{abstract}

Results: In our series, CHCC-CC accounted for $1.6 \%$ of all primary liver malignancy. Mean age of patient with CHCC-CC was 52.8 year (range: 28-74 year) and $88.9 \%$ (40/45) of patients were male. Thirty of forty five patients (66.7\%) had cirrhosis and $20 \%$ (9/45) of patients had chronic hepatitis B without cirrhosis. Alpha-fetoprotein (AFP) was elevated in 62.2 \% (28/45) of patients and carbohydrate antigen 19-9 (CA19-9) elevated in $22.2 \%$ (10/45) of patients). Both AFP and CA19-9 were simultaneously elevated in $15.6 \%$ (7/45) of patients. Enhancement pattern resembling cholangiocarcinoma (CC) was noted in $53.3 \%$ (24/45) of patients (on CEUS and in $30.2 \%$ (13/43) of patients at CT. Enhancement pattern resembling hepatocellular carcinoma (HCC) was observed in $42.2 \%$ (19/45) of patients on CEUS and in $58.1 \%(25 / 43)$ of patients at CT. The percentage of tumors showing CC enhancement pattern $(27.9 \%, 12 / 43)$ was comparable with that of tumors showing HCC enhancement pattern $(44.2 \%, 19 / 43)$ on both CEUS and $\mathrm{CT}(p=0.116)$.

Simultaneous elevation of tumor markers (AFP and CA19-9) or tumor marker elevation (AFP or CA19-9) in discordance with enhancement pattern on CEUS was demonstrated in $51.1 \%(23 / 45)$ of patients and on CT in $53.5 \%$ (23/43) of patients, which was significantly more than simultaneous elevation of tumor markers (AFP and CA19-9) alone $(p=0.000)$.

(Continued on next page)

\footnotetext{
* Correspondence: raylee7991@163.com

'Department Hepato-biliary-Pancreatic Surgery, Southwest Hospital,

Gaotangyan street, Shapingba, Chongqing 400038, P. R China

Full list of author information is available at the end of the article
} 
(Continued from previous page)

Conclusions: The clinical characteristics of CHCC-CC are similar to those of HCC. The CHCC-CC tumors display enhancement patterns resembling CC or HCC in comparable proportion on both CEUS and CT. Combination of simultaneous elevation of tumor makers (AFP and CA19-9) and tumor mark elevation (AFP or CA19-9) in discordance with presumptive imaging findings on CEUS or CT may lead significantly more patients to be suspicious of the diagnosis of CHCC-CC.

Keywords: Combined hepatocellular-cholangiocarcinoma, Contrast-enhanced ultrasound, Computed tomography, Alpha-fetoprotein, Carbohydrate antigen 19-9

\section{Background}

Combined hepatocelluar - cholangiocarcinomas (cHCC$\mathrm{CC}$ ) are uncommon form of primary hepatic carcinoma, accounts for 1.0-6.3\% of all primary liver cancers in Asia and 2.4-14.2 \% of all primary liver cancers in Western countries [1, 2]. It was first defined by Allen and Lisa [3] and has been divided into three types of cHCC-CC. Type A is termed "double cancer" and represents cases in which hepatocelluar carcinoma (HCC) and cholangiocarcinomas (CC) exist separately. Type B, which is called the combined type, is defined as cases in which HCC and CC locates contiguously but independently. The type $\mathrm{C}$, which is referred to as the mixed type, occurs when $\mathrm{HCC}$ and $\mathrm{CC}$ components intermingle within the same tumor. Only Allen and Lisa type C tumors involved a mixture of both hepatocellular and biliary epithelial cell differentiation, appear to be true biphenotypic cHCC-CC. It is postulated that evolution of $\mathrm{CHCC}-\mathrm{CC}$ tumors from common hepatic progenitor cells may account for the biphenotypic features of these tumors [4-8]. Due to the relative rarity of this tumor type, little is known about the risk factors, imaging appearance, or prognosis. Few studies have demonstrated risk factors that overlap with hepatocellular carcinoma (HCC) and cholangiocarcinoma (CC), though not all appear to arise in the background of cirrhosis [9]. The clinical characteristics of cHCC-CC were similar to those of HCC [10], but overall survival was more similar to or poorer than that of CC $[2,10]$. Survival may be related to tumor biology rather than the cause. Multimodal treatment with an initial aggressive therapeutic approach can improve survival [10]. Given the overlap in patient demographics and the unique nature of $\mathrm{CHCC}-\mathrm{CC}$, preoperative diagnosis is crucial for appropriate management. Few studies have evaluated the radiological characteristics of cHCC-CC on computed tomography (CT) or magnetic resonance imaging (MRI) with limited number of patients [11-15]. The presence of imaging features of both HCC and CC in the same tumor may alert the radiologist to the possibility of $\mathrm{CHCC}-\mathrm{CC}$ though which occurred in the minority of cases $[9,15]$. To our knowledge, no study has reported the imaging features of cHCC-CC on contrast-enhanced ultrasound
(CEUS) up to now. The main tumor markers of interest are carbohydrate antigen 19-9(CA19-9) and $\alpha$-fetoprotein (AFP), which are useful adjuncts to imaging in patients with CC and HCC respectively [16]. Simultaneous elevation of both CA 19-9 and AFP has been suggested as highly concerning for cHCC-CC tumors [17]. Other reports suggest that discordance between serum tumor marker elevation and imaging morphology may be suggestive [18]. However, these results were based on clinical data from very limited number of patients and the imaging features of CEUS not included. Therefore, the purpose of this study was to retrospectively evaluate the demographics, clinical presentation, and imaging features on CT and CEUS in patients with cHCC-CC tumors, in the hope of defining features of the uncommon malignant hepatic tumor that may improve preoperative diagnosis and better guide clinical management decisions.

\section{Methods}

\section{Patient population and clinical information}

Institutional review board approval for this study was obtained from the ethics committee of Southwest hospital. The need for informed consent of patient was waived by the ethics committee of Southwest hospital in this retrospective study. From January 2005 to December 2014, pathology databases of our hospital recorded 2863 patients with primary liver cancer, including 46 patients with mixed type (biphenotypic) of $\mathrm{cHCC}-\mathrm{CC}$ which accounted for $1.6 \%$ of all primary liver cancer. One case of cHCC-CC without available CEUS imaging was excluded from the present study. Patients with available cross sectional imaging were included in the study. Clinical information was retrospectively found from our hospital information system. Serum tumor markers reported were drawn before treatment and within 1 week of the imaging examination. Normal values were $0-20 \mathrm{ng} / \mathrm{ml}$ for AFP and $0-22 \mathrm{U} / \mathrm{ml}$ for CA19-9 in our hospital. Cirrhosis was confirmed histopathologically through examination of resected liver specimen.

All the 45 tumors were excised and underwent tissue diagnosis of the surgical specimen. Liver sections were 
examined by pathologists with over 20 year experience of liver pathology (XCY and DYG). The final diagnosis of biphenotypic primary liver carcinoma (both types of tumor intermixed) depended on a combination of $\mathrm{H}$ and $\mathrm{E}$ stain findings [19] and proof of both hepatocellular (polyclonal arcinoembryonic antigen, a marker of canalicular formation,) and biliary differentiation (keratin 7 or keratin19) immunohistochemical markers. Sub-classification was done according o the 2010 World Health Organization Tumors of the Digestive System classification [20]. In patient with more than one lesion,the pathological diagnosis of the largest one was confirmed biphenotypic cHCC-CC. Another 3 patients with double cancer (HCC and CC exist separately) and 7 patients with combined tumors (both types of tumor contiguous to each other) were excluded from the present study.

We performed a per-patient analysis. In a patient with more than one solid lesion in the liver, only the largest one was measured and investigated because CEUS could not scan multiple nodules simultaneously after one injection of contrast agent if the nodules are not at the same scan plane and it is difficult to correspond the pathology of each tumor to the imaging of US in patients with multiple hepatic lesions.

\section{US scan}

US examinations were performed by two experienced physicians with over 16 years of experience of liver ultrasound examination ( $\mathrm{R} \mathrm{L}$, and $\mathrm{XH} \mathrm{Z}$ ) with an Acuson Sequoia 512 ultrasound unit (Siemens Medical Solutions, Santa Clara, Calif). Baseline US was performed with a multifrequency $4 \mathrm{C} 1$ convex array probe. The gray-scale US characteristics of the lesion, including location, size, shape, and echogenicity were recorded. CEUS was performed by using contrast pulse sequencing (CPS) imaging. Real-time contrast imaging setting was used with a low mechanical index of $<0.2$ and a volume of $2.4 \mathrm{~mL}$ of blood pool contrast agent (SonoVue, Bracco Imaging B.V, Geneva, Switzerland) was injected into cubital vein in bolus via a 20-gauge needle followed by a $5 \mathrm{~mL}$ saline flush. After contrast medium injection, hepatic lesion was scanned continuously for up to $4 \mathrm{~min}$. The whole vascular phase was studied, consisting of the arterial phase $(0-30 \mathrm{~s}$ from beginning of contrast agent bolus injection), portal phase (31-120 s after the injection), and delayed phase (121-240 s after the injection) according to EFSUMB recommendations [21]. All ultrasound images of cHCC-CC tumors were reviewed retrospectively for this study by two physicians ( $\mathrm{R} \mathrm{L}$, and $\mathrm{XH} \mathrm{Z}$ ) in consensus.

\section{CEUS image analysis}

The contrast vascular patterns on CEUS were defined by comparing the enhancement behaviour of the tumor with the surrounding liver parenchyma and classified as:

(1) Peripheral hyperenhancement-irregular rim-like hyperenhancement at the peripheral part of the lesion with sparse filiform and punctiform internal enhancement.

(2) Heterogeneous hyperenhancement-when the lesion displays mixed hyperenhancement inhomogeneously at both the periphery and the central part of the lesion.

(3) Homogeneous hyperenhancement-when the whole lesion shows hyperenhancement homogeneously.

(4) Hypoenhancement-the lesion enhances in the less degree than that of the surrounding liver parenchyma.

(5) Isoenhancement-the lesion enhances in the similar degree as the surrounding liver parenchyma.

(6) Non-enhancement-no appearance of contrast agent (microbubbles) at both the periphery and the central part of the lesion.

Wash-out appearance was considered as the presence of hypoenhacement of the lesion in the portal or late phases preceded by hyperenhancement in the arterial phase. A lesion lacking any enhancement in all phases was not defined as washout. When a nodule shows heterogeneous hyperenhancement in the arterial phase, observation of washout was confined to the area showing hyperenhancement in the arterial phase, and the area showing non-enhancement was excluded from the observation. The extent of washout was classified as:

(1) Marked washout-when the lesion displays obviously lower echogenicity than the surrounding liver parenchyma in the portal or late phases preceded by hyperenhancement in the arterial phase.

(2) Mild washout - when the lesion shows slightly lower echogenicity than the surrounding liver parenchyma in the portal or late phases preceded by hyperenhancement in the arterial phase.

(3) No washout-when the lesion enhances in the similar degree or higher degree as the surrounding liver parenchyma in the portal or late phases preceded by hyperenhancement in the arterial phase.

Typical HCC pattern on CEUS was defined as hyperenhancement in the arterial phase followed by slow or slight washout in the portal or late phase,and the wash out tends to start later in HCC, usually not before $60 \mathrm{~s}$ after injection [22, 23]. On the contrary, most peripheral cholangiocarcinoma showed wash out emergence before 
$60 \mathrm{~s}$ and marked wash out in the portal phase on CEUS $[24,25]$. Typical CC pattern on CEUS was defined as: (1) Peripheral arterial rim enhancement followed by marked washout in the portal phase; (2) Arterial hyperenhancement followed by quick (washout begins before $60 \mathrm{~s}$ after contrast agent injection) and marked washout in the portal phase [22, 24, 25].

\section{CT scan}

Abdomen CT was performed with multidetector-row CT (MDCT, 64 detector rows, Definition, Siemens, Erlangen, Germany) using a 4 phase contras-enhanced protocol (unenhanced, hepatic arterial, portal venous, and delayed phases). First, an unenhanced scan was obtained through the liver. Next, after intravenous infusion of $2 \mathrm{ml} / \mathrm{kg}$ of a nonionic iodine-containing contrast agent (ultravist 370, Scherning AG, Berlin, Germany) using a power injector (Stellant CT Injection System, Medrad, Indianola, Pennsylvania) at a rate of $4.0 \mathrm{ml} / \mathrm{s}$, contrast-enhanced scans were obtained in arterial with bolus test trigger for optimal characterization of focal hepatic lesions. Data acquisitions were obtained through the whole liver in a craniocaudal direction during a single breath-hold helical acquisition for 4-6 s with $5 \mathrm{~mm}$ slice thickness and $0.5 \mathrm{~s}$ rotation time. The acquisition of the arterial phase was automatically started $5 \mathrm{~s}$ after contrast agent reaching the threshold in the aorta. The start of acquisition sequences was $60 \mathrm{~s}$ for the portal venous phase and $180 \mathrm{~s}$ for the delayed phase.

\section{Categorization of enhancement patterns at CT}

The enhancement through each of the different phases after intravenous contrast administration was registered as follows: (1) globally hyperdense: increased signal relative to the surrounding liver parenchyma, involving the totality of the lesion; (2) partially hyperdense: increased signal involving more than $50 \%$ of the lesion crosssection area with a non-homogeneous distribution; (3) peripherally hyperdense: increased signal limited to the periphery of the lesion, involving less than $25 \%$ of its area, resembling a rim-like pattern; (4) isodense: same density as the surrounding liver parenchyma; (5) hypodense: lower density compared to the liver parenchyma involving more than half of the cross-sectional area of the tumor. Dynamic pattern of enhancement was defined according to the analysis of the progression of contrast enhancement over different phases of the study, as follows: (1) stable or persistent contrast enhancement: the nodule enhancement is unmodified from the arterial to the portal venous and delayed phases; (2) progressive contrast enhancement: the nodule enhances progressively over time, reaching maximal intensity in delayed phases; (3) 'wash-out' pattern: global intense/partial hyperdense of the lesion during the arterial phase followed by hypodense in portal and/or delayed venous phases; (4) all other cases. This classification was adopted from Rimola et al. [26] and lavarone et al. [27].

CT findings were evaluated in consensus by 2 abdominal radiologists (PC and SYD) with over 15 years of experience in liver radiology who were blinded to CEUS findings and pathological results of the tumors. Typical HCC pattern on CT was defined as hyperenhancement in the arterial phase followed by washout in the portal or late phase of the nodule [28]. Typical CC pattern on $\mathrm{CT}$ was defined as progressive centripetal enhancement or stable persistent enhancement of the nodule [27].

\section{Statistical analysis}

We performed a per-patient analysis in this study. Characteristics of the patients are expressed as median and range or count and proportion. Comparison of CEUS and $\mathrm{CT}$ was done by using the chi-squared test for categorical variables. A $P$ value of less than 0.05 was considered statistically significant. Statistical analysis was performed using the SPSS 13.0 software package (SPSS Inc, Chicago, IL).

\section{Results}

\section{Clinicopathologic features}

Demographics of 45 patients with cHCC-CC are shown in Table 1. Mean age of patients was 52.8 year (range: $28-74$ year) and 40 patients were male $(88.9 \%)$. Thirty patients $(66.7 \%)$ had cirrhosis. The etiology of cirrhosis was viral hepatitis B infection in 24 patients, combination of viral hepatitis $B$ infection and alcohol misuse in 5 patients. Nine patients had chronic hepatitis B without cirrhosis $(20 \%)$. Of the 45 patients with cHCC-CC tumors, 6 presented incidentally, 10 discovered on cirrhosis screening, 22 presented with abdominal pain, 2 with jaundice, 2 with tarry stool, 1 with edema of lower limbs. Of the 22 patients presented with abdominal pain, 4 patients had a palpable mass. In two patients, the presentation is unknown. All the 45 patients had AFP assay which was abnormally elevated in 28 patients $(62.2 \%)$ and normal in 17 patients. Forty three patients had serum assays of carcinoembryonic antigen (CEA) which was elevated in 5 patients $(11.6 \%)$ and normal in 38 patients. Forty two patients had serum assays of CA-125 which was elevated in 6 patients $(14.3 \%)$ and normal in 36 patients. Forty five patients had serum assays of CA19-9 which was elevated in 10 patients $(22.2 \%)$ and normal in 35 patients. Both AFP and CA19-9 were simultaneously elevated in 7 patients (15.6\%). The average size of cHCC-CC was $5.3 \mathrm{~cm}$ and over half of them were less than $5 \mathrm{~cm}$. Most patients had single tumor and over half of them were located in the right lobe of liver. In 6 patients with 2 hepatic nodules, pathological diagnosis of all the nodules was cHCC-CC. In 1 patient with 4 
Table 1 Demographics of 45 patients with combined hepatocellular-cholangiocarcinoma

\begin{tabular}{|c|c|c|c|}
\hline & Number (\%) & Mean \pm SD & Median (range) \\
\hline Age (year) & & $52.8 \pm 10.4$ & $51.5(28-74)$ \\
\hline Male/female & $40 / 5$ & & \\
\hline $\operatorname{AFP}(n g / m l)$ & & $680.8 \pm 1700.2$ & $68.6(1.02-9675)$ \\
\hline$\leq 20$ & $17(37.8)$ & & \\
\hline $21-200$ & $15(33.3)$ & & \\
\hline$>200$ & $13(28.9)$ & & \\
\hline \multicolumn{4}{|l|}{ Underling liver disease } \\
\hline Cirrhosis & $30(66.7)$ & & \\
\hline Chronic hepatitis B & $4(8.9)$ & & \\
\hline \multicolumn{4}{|l|}{ Chronic hepatitis C } \\
\hline Alcoholic & $3(6.7)$ & & \\
\hline Chronic hepatitis B and alcoholic & $5(11.1)$ & & \\
\hline No evidence of chronic liver disease & $3(6.7)$ & & \\
\hline Nodule size $(\mathrm{cm})$ & & $5.3 \pm 3.2$ & $4.5(1.5-13.8)$ \\
\hline$<5 \mathrm{~cm}$ & $24(53.3)$ & & \\
\hline $5-10 \mathrm{~cm}$ & $15(33.3)$ & & \\
\hline$>10 \mathrm{~cm}$ & $6(13.3)$ & & \\
\hline \multicolumn{4}{|l|}{ Number of nodules } \\
\hline Single & $38(84.4)$ & & \\
\hline Two & $6(13.3)$ & & \\
\hline More than three & $1(2.2)$ & & \\
\hline \multicolumn{4}{|l|}{ Location of nodules } \\
\hline Left lobe & $8(17.8)$ & & \\
\hline Right lobe & $31(68.9)$ & & \\
\hline Both left and right lobe & $3(6.7)$ & & \\
\hline Caudate lobe & $3(6.7)$ & & \\
\hline \multicolumn{4}{|l|}{ Echogenicity of nodules } \\
\hline Hypoechoic & $23(51.1)$ & & \\
\hline Isoechoic & $2(4.4)$ & & \\
\hline Hyperechoic & $12(26.7)$ & & \\
\hline Mixed & $8(17.8)$ & & \\
\hline
\end{tabular}

hepatic nodules, pathological diagnosis of 3 nodules, including the largest one was cHCC-CC, and 1noule was heamangioma.

\section{Radiological features}

The enhancement appearances of cHCC-CC on CEUS in 45 patients are shown in Table 2. Tumors showing peripheral hyperenhancement in the arterial phase followed by marked washout in the portal phase in 9 patients were defined as CC pattern. Tumors showing heterogeneous (8 tumors) or homogeneous (7 tumors) hyperenhancement in the arterial phase followed by early (washout begins earlier than $60 \mathrm{~s}$ ) and marked wash out during the portal phase were judged as CC pattern. Tumors showing heterogeneous (13 tumors) or homogeneous (6 tumors) hyperenhancement in the arterial phase followed by both slow (washout begins later than $60 \mathrm{~s}$ ) and mild wash out in the portal or late phase were judged as HCC pattern. The enhancement pattern of 2 patients was judged as indeterminate because one tumor showed heterogeneous hyperenhancemen in the arterial phase followed by isoenhancement in the portal and the late phase, and another tumor displayed hypoenhancement in the arterial phase, remained hypoenhancement in the portal and the late phase. The average time of washout emergence on CEUS was $58.18 \mathrm{~s}$ (median :53 s, range :22 s $\sim 129 \mathrm{~s}$ ). The percentage of tumors showing CC enhancement pattern (53.3\%) was similar 
Table 2 The enhancement patterns of combined hepatocellular-cholangiocarcinoma on CEUS in 45 patients

\begin{tabular}{|c|c|}
\hline Enhancement appearance & Number $\%$ \\
\hline \multicolumn{2}{|l|}{ Arterial phase } \\
\hline Peripheral hyperenhancement & $9(20.0)$ \\
\hline Heterogeneous hyperenhancemen & $22(48.9)$ \\
\hline Homogeneous hyperenhancement & $13(28.9)$ \\
\hline Hypoenhancement & $1(0.2)$ \\
\hline Isoenhancement & $0(0)$ \\
\hline \multicolumn{2}{|l|}{ Portal phase } \\
\hline \multicolumn{2}{|l|}{ Peripheral hyperenhancement } \\
\hline Heterogeneous hyperenhancement & $1(0.2)$ \\
\hline Homogeneous hyperenhancement & $0(0)$ \\
\hline Slight hypoenhancement & $17(37.8)$ \\
\hline Marked hypoenhancement & $25(55.6)$ \\
\hline Isoenhancement & $2(0.4)$ \\
\hline \multicolumn{2}{|l|}{ Delayed phase } \\
\hline \multicolumn{2}{|l|}{ Peripheral hyperenhancement } \\
\hline Heterogeneous hyperenhancement & $1(0.2)$ \\
\hline \multicolumn{2}{|l|}{ Homogeneous hyperenhancement } \\
\hline Slight hypoenhancement & $8(17.8)$ \\
\hline Marked hypoenhancement & $35(77.8)$ \\
\hline Isoenhancement & $1(0.2)$ \\
\hline \multicolumn{2}{|l|}{ Emergence of-washout } \\
\hline$<60$ seconds & $28(62.2)$ \\
\hline $60-120$ seconds & $13(28.9)$ \\
\hline$>120$ seconds & $2(4.4)$ \\
\hline No washout & $2(4.4)$ \\
\hline
\end{tabular}

to that of tumors showing $\mathrm{HCC}$ enhancement pattern (42.2\%) on CEUS ( $p=0.291)$.

Forty three patients had unenhanced CT scan. Forty of the tumors were hypodense and 3 were hyperdense. Hepatic capsular retraction was revealed in 4 cases $(9.3 \%)$. Three patients had intrahepatic biliary dilatation (7.0\%). Five patients had malignant portal veins thrombus (11.6\%) and 5 had regional lymphadenopathy (11.6\%). Intrahepatic metastasis was observed in 7 patients (16.3\%). Enhancement appearances of cHCC-CC on contrastenhanced CT in 43 patients are shown in Table 3. Tumors showing stable persistent peripherally hyperenhancement from the arterial phase to the late phase in 8 patients were defined as CC pattern. Tumors showing progressive delayed enhancement from the arterial phase to the late phase in 5 patients were also judged as CC pattern. Tumors showing hyperenhancement (24 heterogeneous, 1 homogeneous) in the arterial phase followed by washout in the portal or the late phase in 25 patients were defined as HCC pattern. Tumors showing hypoenhancement from the arterial phase to the late phase in 4 patients and 1 tumor showing hyperenhancement in the arterial phase and the portal phase followed by isoenhancement in the late phase were judged as indeterminate pattern. The percentage of tumors showing CC enhancement pattern (30.2\%) was less than that of tumors showing HCC enhancement pattern $(58.1 \%)$ on CT $(p=0.009)$. Twelve of forty three patients displayed CC enhancement pattern on both CEUS and CT (27.9\%), while $44.2 \%$ (19/43) of patients demonstrated HCC enhancement pattern at both CEUS and CT. The percentage of tumors showing CC enhancement pattern was comparable with that of tumors showing HCC enhancement pattern $(p=0.116)$.

\section{Correlations of AFP and CA19-9 levels with enhancement patterns on CEUS and CT}

In 7 patients with simultaneous elevation of both AFP and CA19-9, CC enhancement pattern was observed in 5 patients on CEUS and 1 patient at CT. HCC enhancement pattern was noted in 2 patients on CEUS and 6 patients at $\mathrm{CT}$ respectively (Fig. 1). Correlations between AFP test and enhancement patterns of CEUS and CT 
Table 3 Enhancement patterns of combined hepatocellularcholangiocarcinoma on $\mathrm{CT}$ in 43 patients

\begin{tabular}{lccc}
\hline & $\begin{array}{c}\text { Arterial } \\
\text { phase }\end{array}$ & $\begin{array}{c}\text { Portal } \\
\text { phase }\end{array}$ & $\begin{array}{c}\text { Delayed } \\
\text { phase }\end{array}$ \\
\hline $\begin{array}{l}\text { Peripherally hyperdense } \\
\text { No.(\%) }\end{array}$ & $12(27.9)$ & $13(30.2)$ & $7(16.3)$ \\
$\begin{array}{l}\text { Partially hyperdense No.(\%) } \\
\text { Globally hyperdense No.(\%) }\end{array}$ & $24(55.8)$ & $5(11.6)$ & $6(13.9)$ \\
$\begin{array}{l}\text { Isodense No.(\%) } \\
\text { Hypodense No.(\%) }\end{array}$ & $5(11.6)$ & $25(58.1)$ & $29(67.4)$ \\
\hline
\end{tabular}

are shown in Table 4. AFP was elevated and CA19-9 normal in 9 of $45(20.0 \%)$ patients showing CC enhancement pattern on CEUS and in 7of 43 (16.3\%) patients showing CC enhancement pattern on CT $(p=$ 0.651) (Fig. 2). In 3 patients with elevated CA19-9 and normal AFP, HCC enhancement pattern was noted in 3 patients on CEUS and in 2 patients at CT (Fig. 3), $\mathrm{CC}$ enhancement pattern was observed 1 patient at CT. Correlations between CA19-9 test and enhancement patterns of CEUS and CT are shown in Table 5. Elevated tumor markers (AFP or CA19-9) were in discordance with imaging findings in 19 of 45 (42.2\%) patients on CEUS and in 16 of 43 (37.2\%) patients at CT $(p=0.0 .631)$. Simultaneous elevation of tumor markers (AFP and CA19-9) or tumor marker elevation (AFP or CA19-9) in discordance with enhancement pattern on CEUS was demonstrated in 26 of 45 patients, which was significantly more than simultaneous elevation of tumor markers (AFP and CA19-9) alone (7/ $45, p=0.000)$. Simultaneous elevation of tumor markers (AFP and CA19-9) or tumor marker elevation (AFP or CA19-9) in discordance with enhancement pattern on CT was observed in 23 of 43 patients, which was significantly more than simultaneous elevation of tumor markers (AFP and CA19-9) alone $(7 / 43, p=0.000)$.

\section{Discussion}

Combined hepatocellular-cholangiocarcinoma accounts for $0.4-14.2 \%$ of all primary liver carcinomas, with not only local incidence varying considerably between regions $[16,18]$, but also the different including criteria for classification in previous literature. Although some published reports involving only Allen and Lisa type $\mathrm{C}$ of cHCC-CC $[18,29]$, others either included all three types of $\mathrm{cHCC}-\mathrm{CC}[30,31]$ or did not state their criteria for diagnosis and classification [32, 33]. From January 2005 to December 2014, 46 out of 2863 patients underwent surgery or biopsy for hepatic malignancy at our hospital had unequivocal mixed type (biphenotypic) of cHCC$\mathrm{CC}$. The incidence of this real mixed type cHCC-CC composed of cholangiocyte-derived and hepatocytederived neoplastic elements is $1.6 \%$. In the present series, most patients with $\mathrm{cHCC}-\mathrm{CC}$ had cirrhosis (66.7 \%) or chronic hepatitis B (20\%). The mean age of patients was 52.8 year and $88.9 \%$ of patients were male. Our data demonstrated that $\mathrm{CHCC}-\mathrm{CC}$ developed more

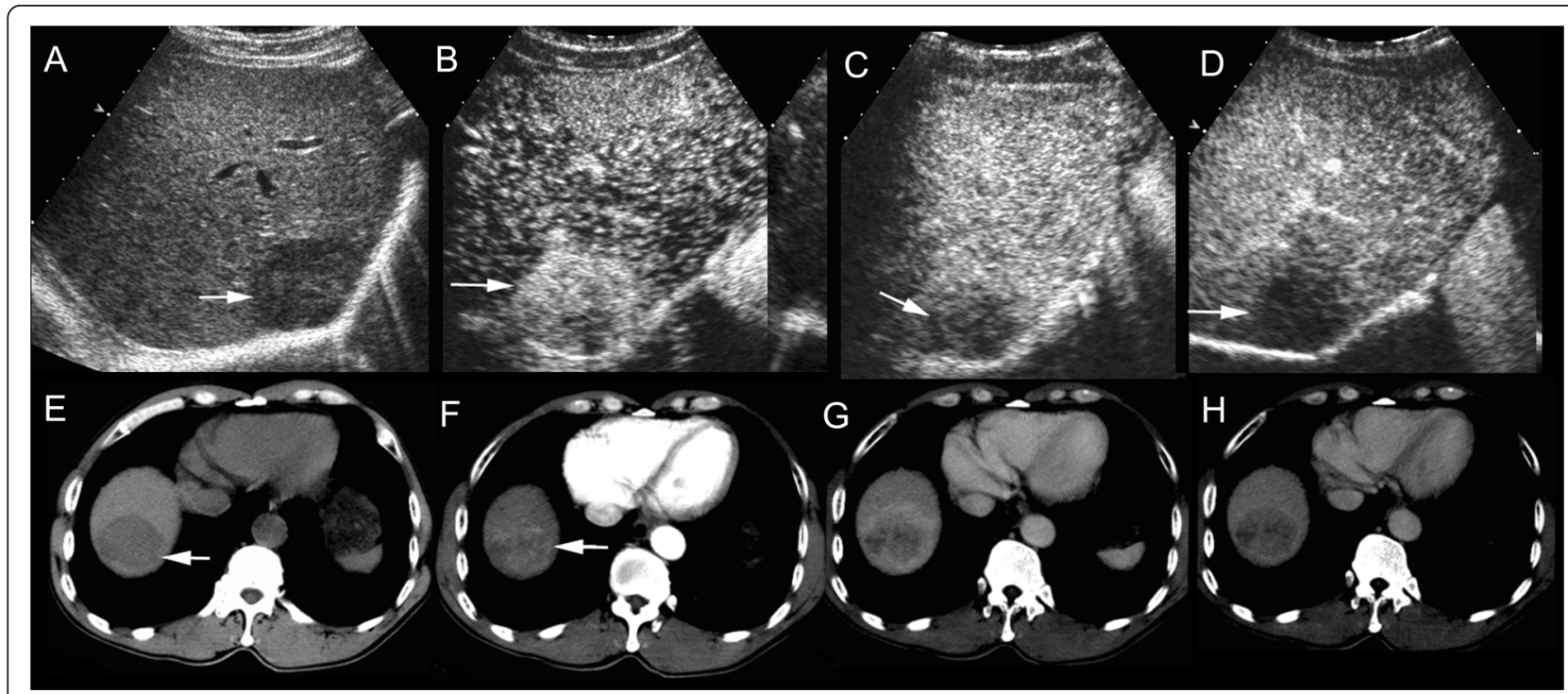

Fig. 1 CHCC-CC in a patient with chronic hepatitis B. Simultaneous elevation of AFP (718 ng/ml) and CA19-9 (45.0U/ml) was detected in the patient. Unenhanced ultrasound shows a hypoechoic mass of $4.6 \mathrm{~cm}$ in right lobe of the liver ( $\mathbf{a}$, arrow). The mass displays heterogeneous hyperenhancement in the arterial phase (b, $26 \mathrm{~s}$ after contrast agent injection) followed by quick (c, $58 \mathrm{~s}$ after injection) and marked washout (d, $102 \mathrm{~s}$ after injection) in the portal phase on CEUS, resembling the enhancement pattern of intrahepatic cholangiocarcinoma. Unenhanced CT scan reveals a hypodense mass in right lobe of the liver (e, arrow). The mass displays heterogeneous hyperenhancemen in the arterial phase (f, arrow) followed by washout (g) in the portal phase and the late phase (h) on contrast-enhanced $C$, resembling the enhancement pattern of HCC 
Table 4 Correlation between AFP test and enhancement patterns of CEUS and CT

\begin{tabular}{lcccccccc}
\hline & \multicolumn{3}{c}{ CEUS pattern $(n=45)$} & & \multicolumn{3}{c}{ CT pattern $(n=43)$} \\
\cline { 2 - 3 } & CC & HCC & indeterminate & & CC & HCC & indeterminate \\
\hline AFP normal & 10 & 7 & & & 5 & 9 & 3 \\
AFP elevated & 14 & 12 & 2 & & 8 & 16 & 2 \\
\hline
\end{tabular}

frequently in a middle-aged male population with chronic hepatitis and cirrhosis mostly related to chronic hepatitis $\mathrm{B}$, indicating the clinical characteristics of $\mathrm{cHCC}-\mathrm{CC}$ are similar to those of HCC $[10,34]$. However, this is inconsistent with two reports from Western countries [15, 18], which published data of 27 patients and 29 patients, cirrhosis was seen in $0 \%$ and $20 \%$, positive hepatitis B or C detected in $15 \%$ and $10 \%$ of the patients respectively. The discrepancy may relate to different population background characteristics, reflecting the high rates of hepatitis B viral infection in Chinese populations. Although the majority of patients had insidious onset of cHCC-CC with late presentation of advanced disease, $35.6 \%$ of our patients were discovered on cirrhosis screening or presented incidentally at early stage for regular health examination by ultrasound.

In our study, $27.9 \%$ of the cases showed arterial peripheral hyperenhancement on CT, which is lower than previous reports by Ebied et al. (50 \%) and Fowler et al. (51.9\%) $[13,15]$. On the contrary, $60.5 \%$ of our patients displayed heterogeneous or homogeneous hyperenhancement in the arterial phase that is higher than in the study by Ebied et al. (33.3\%). Patients with $\mathrm{cHCC}-\mathrm{CC}$ in the present study demonstrated more HCC enhancement pattern (58.1\%) and less CC enhancement pattern (30.2\%) than reported by Fowler et al. (31.0 \%, 41.4 \% respectively). An explanation of these discrepancies may be the tumor size, which was much smaller in our study (median size $4.5 \mathrm{~cm}$ ) as compared with the data reported by Ebied et al. (median size $7 \mathrm{~cm}$ ) and by Fowler et al. (median size $7.5 \mathrm{~cm}$ ). As the tumor grows larger, a relatively smaller blood supply is available, leading to necrosis and more fibrous stroma formation in the central portion of the tumor, which constitute the pathological background of CC enhancement pattern on contrast-enhanced CT [35]. Capsular retraction and biliary ductal dilatation have been considered important ancillary features of $\mathrm{CC}$. Less patients with cHCC-CC revealed capsular retraction (9.3\%) and biliary ductal dilatation (7.0\%) on CT in our data than reported by Ebied et al. (26.7 \%, $16.7 \%)$ and by Fowler et al. (41.4 \%, $34.5 \%)$. We favor to interpret these inconformity in the light of the differences in tumor size and the underling liver diseases, namely, the tumors of our patients were much smaller and more patients had cirrhosis than in the previous series mentioned above.

Imaging characteristics of $\mathrm{CHCC}-\mathrm{CC}$ on CEUS has not been reported up to now. Our study demonstrated that $95.6 \%$ of the tumor showed washout enhancement pattern on CEUS, indicating a malignant nature of the tumor. Imaging features of $\mathrm{cHCC}-\mathrm{CC}$ may display as $\mathrm{CC}$

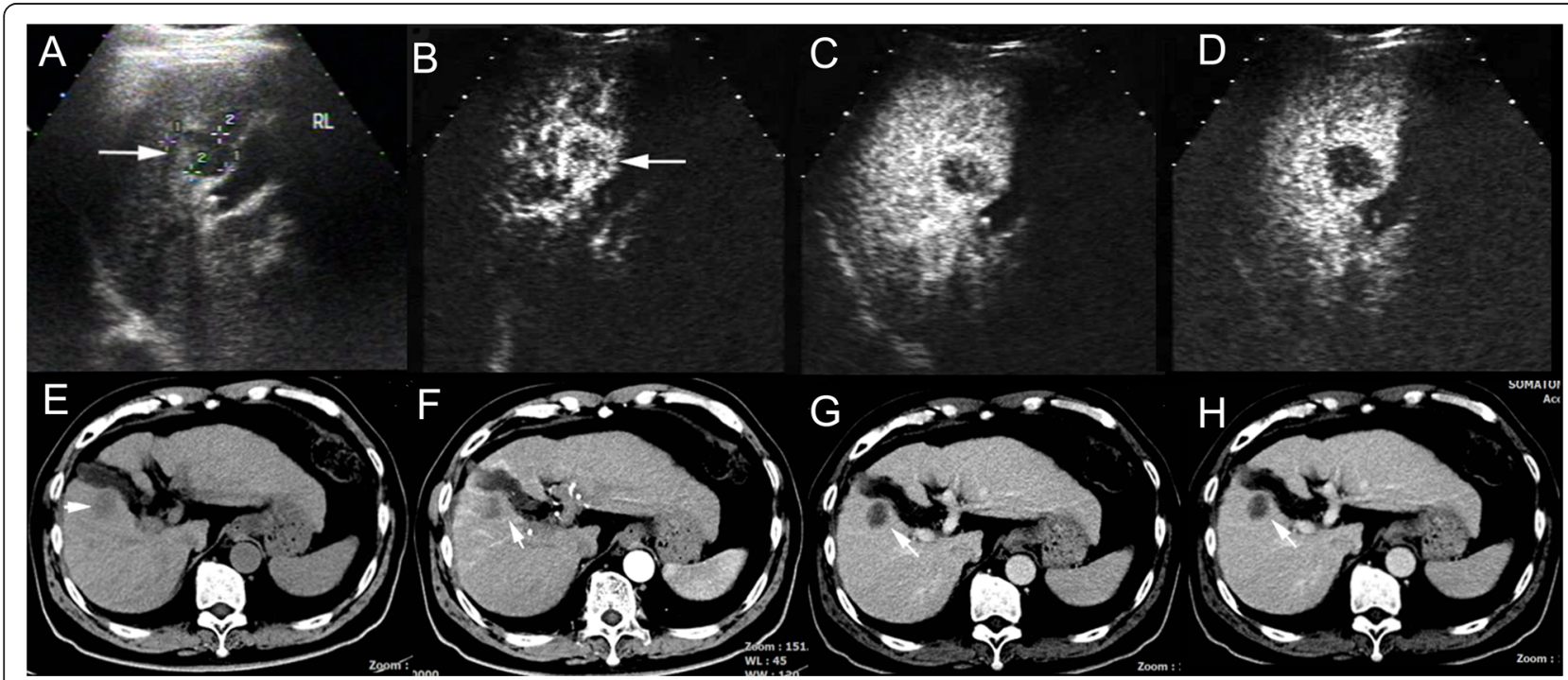

Fig. 2 CHCC-CC in a patient with cirrhosis related to chronic hepatitis B infection. CA19-9 test was normal and AFP elevated (178.5 ng/ml) in discordance with presumptive imaging findings in the patient. Unenhanced ultrasound shows a hypoechoic nodule (a, arrow) of $2.7 \mathrm{~cm}$ in right lobe of the liver (RL). The nodule demonstrates peripheral rim-like enhancement in the arterial phase (b, $18 \mathrm{~s}$ after contrast agent injection, arrow) followed by quick (c, $37 \mathrm{~s}$ after injection) and marked washout (d, $82 \mathrm{~s}$ after injection) in the portal phase on CEUS, resembling the enhancement pattern of intrahepatic cholangiocarcinoma. Unenhanced CT scan reveals a hypodense nodule in right lobe of the liver (e, arrow). The nodule displays peripheral rim-like enhancement in the arterial phase (f, arrow) followed by stable and persistent peripheral rim-like enhancement in the portal ( $\mathbf{g}$ ) and the late phase (h, arrow), resembling the enhancement pattern of intrahepatic cholangiocarcinoma 


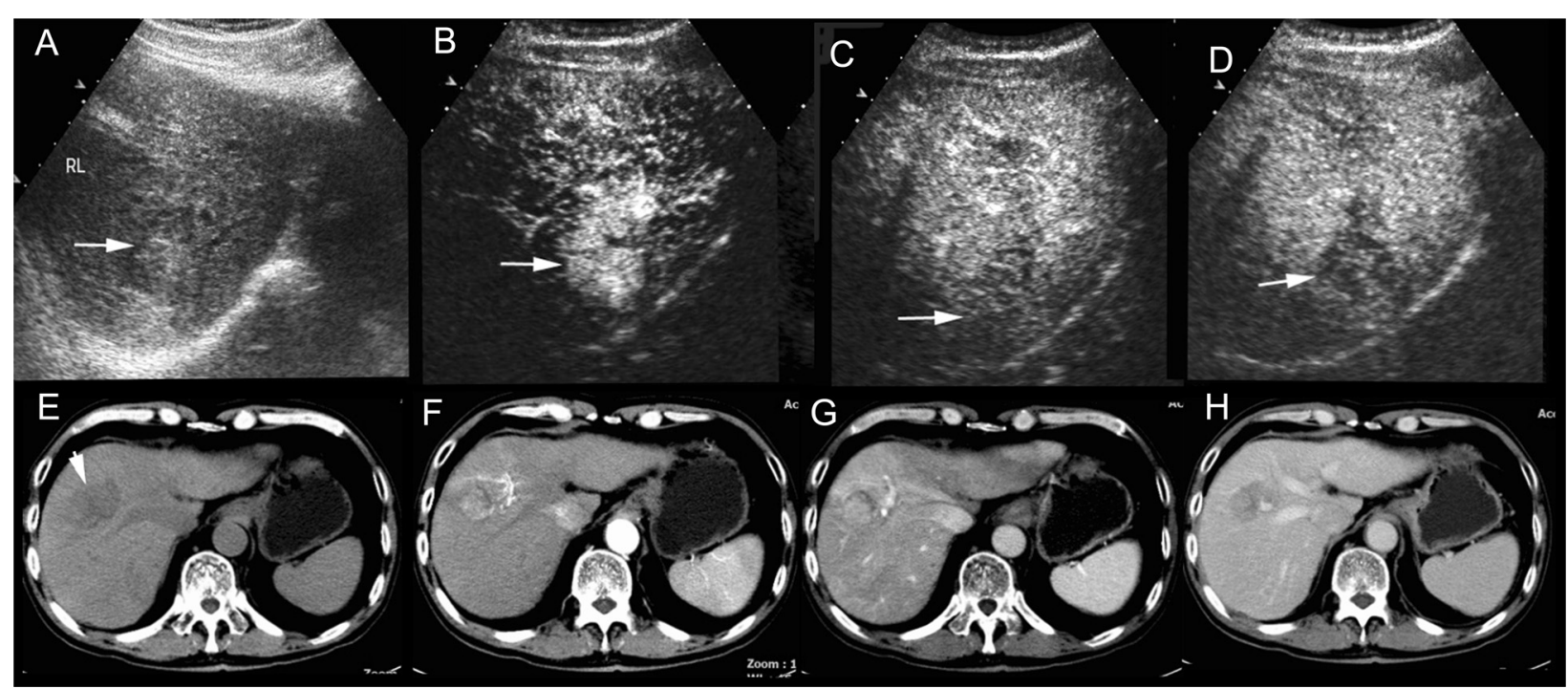

Fig. 3 CHCC-CC in a patient with cirrhosis related to chronic hepatitis B infection. AFP test was normal and CA19-9 elevated (50.3U/ml) in discordance with presumptive imaging findings in the patient. Unenhanced ultrasound shows a hyperchoic mass of $3.7 \mathrm{~cm}$ with hypoechoic halo (arrow) in right lobe of the liver (a). The mass displays heterogeneous hyperenhancemen in the arterial phase (b, 29 s after contrast agent injection) followed by slow washout in the portal phase (c, $110 \mathrm{~s}$ after injection, arrow) and mild washout (d, $179 \mathrm{~s}$ after injection, arrow) in the late phase on CEUS, resembling the enhancement pattern of HCC. Unenhanced CT scan reveals a hypodense mass in right lobe of the liver (e, arrow). The mass displays heterogeneous hyperenhancement in the arterial phase (f) and the portal phase (g) followed by washout in the late phase $(\mathbf{h})$ on contrast-enhanced $C T$, resembling the enhancement pattern of HCC

enhancement pattern or HCC enhancement pattern. The percentage of the two types of enhancement pattern on CEUS showed no statistical difference in our series. Our results demonstrated that imaging features of $\mathrm{cHCC}-\mathrm{CC}$ may resemble either CC or HCC.CC enhancement pattern and $\mathrm{HCC}$ enhancement pattern are likely present in a comparable proportion in patients with $\mathrm{CHCC}-\mathrm{CC}$ on CEUS.

Serum tumor markers of potential utility in $\mathrm{CHCC}-\mathrm{CC}$ are CA 19-9 and AFP, which are associated with CC and $\mathrm{HCC}$ respectively. When both are simultaneously elevated or elevated in discordance with presumptive imaging findings (i.e., elevated CA 19-9 with imaging findings of $\mathrm{HCC}$ pattern, or elevated AFP with imaging findings of $\mathrm{CC}$ pattern), cHCC-CC should at least be suggested $[9,17]$. However, this point of view was based on few studies with very limited number of patients (less than 15 patients) $[11,17,36]$ and needs to be evaluated in more patients. In addition, the imaging features of cHCC-CC on CEUS have not been taken into consideration up to now. Previous reports demonstrated that

Table 5 Correlation between CA19-9 test and enhancement patterns of CEUS and CT

\begin{tabular}{lccccccccc}
\hline & \multicolumn{3}{c}{ CEUS pattern $(n=45)$} & & \multicolumn{3}{c}{ CT pattern $(n=43)$} \\
\cline { 2 - 3 } & CC & HCC & indeterminate & & CC & HCC & indeterminate \\
\hline CA19-9 normal & 19 & 14 & 2 & & 11 & 17 & 5 \\
CA19-9 elevated & 5 & 5 & & & 2 & 8 & \\
\hline
\end{tabular}

elevated serum AFP levels were found in $33 \%-78 \%$ and elevated CA19-9 in $20 \%-36 \%$ of patients with cHCCCC $[11,17,36]$. In our series, AFP was abnormally elevated in $62.2 \%$ and CA19-9 in $22.2 \%$ of patients, which is comparable to previous reports. AFP and CA19-9 were simultaneously elevated in $15.6 \%$ of patients in the present study, indicating a much low sensibility if this criterion alone was used for suggestion of cHCC-CC. In our study, simultaneous elevation of tumor markers (AFP and CA19-9) or tumor marker elevation (AFP or CA19-9) in discordance with enhancement pattern on CEUS or on CT was demonstrated in significantly more patients $(51.1 \%, 53.3 \%$ respectively) than simultaneous elevation of tumor markers (AFP and CA19-9) alone $(15.6 \%, p=0.000)$, indicating that when both the results of tumor makers and imaging features of CEUS or CT were taken into consideration, the possibility of cHCC$\mathrm{CC}$ may be suggested in significantly more patients.

There are some limitations in the present study. First, it is a retrospective analysis, though the rarity of cHCC$\mathrm{CC}$ lends itself almost exclusively retrospective design. Second, the patient population is relatively small, consisting of 45 patients with cHCC-CC out of 2863 cases of primary liver malignancy. Nevertheless, our sample size is relatively larger than most previously published series. Third, only 2 patients in our study had MRI examination before operation, the imaging features of cHCCCC on MRI could not be summarized. This reflects the fact that in many countries worldwide, with a high 
incidence of HCC, the availability of MRI scan for the diagnosis of focal liver lesions is still much low.

\section{Conclusions}

Combined hepatocelluar - cholangiocarcinomas (biphenotypic) is an uncommon primary liver malignancy with background population characteristics similar to HCC. Imaging features of $\mathrm{CC}$ or $\mathrm{HCC}$ presents in comparable proportion in $\mathrm{CHCC}-\mathrm{CC}$ on both CEUS and CT. Combination of simultaneous elevation of tumor makers (AFP and CA19-9) and tumor mark elevation in discordance with presumptive imaging findings on CEUS or CT may lead significantly more patients to be suspicious of the diagnosis of $\mathrm{CHCC}-\mathrm{CC}$.

\section{Abbreviations \\ CHCC-CC: Combined hepatocelluar - cholangiocarcinomas; HCC: Hepatocelluar carcinoma; CC: Cholangiocarcinomas; CT: Computed tomography; MRI: Magnetic resonance imaging; CEUS: Contrast-enhanced ultrasound; CA19-9: Carbohydrate antigen 19-9; AFP: a -fetoprotein.}

\section{Competing interests}

The authors declare that they have no competing interests.

\section{Authors' contributions}

RL designed, planned and coordinated the study, interpreted the results of CEUS, drafted the manuscript and revised it. CLT collected CEUS data performed the quantitative analysis of CEUS results. XHZ interpreted CEUS images. SYD interpreted images of CT. PC interpreted images of CT. KSM interpreted clinical data, performed liver surgery. DY Collected CT data and clinical data, performed the quantitative analysis of $C T$ and clinical data. DYG interpreted the pathology of CHCC-CC tumor. XCY interpreted the pathology of CHCC-CC tumor. All authors read and approved the final manuscript.

\section{Author details}

'Department Hepato-biliary-Pancreatic Surgery, Southwest Hospital, Gaotangyan street, Shapingba, Chongqing 400038, P. R China. ${ }^{2}$ Department Ultrasound, Southwest Hospital Affiliated to Third Military Medical University, Shapingba, Chongqing 400038, P. R China. ${ }^{3}$ Department Radiology, Southwest Hospital Affiliated to Third Military Medical University, Shapingba, Chongqing 400038, P. R China. ${ }^{4}$ Department Pathology, Southwest Hospital Affiliated to Third Military Medical University, Shapingba, Chongqing 400038, P. R China.

Received: 19 November 2015 Accepted: 10 February 2016 Published online: 25 February 2016

\section{References}

1. Lee WS, Lee KW, Heo JS, Kim SJ, Choi SH, Kim YI, Joh JW. Comparison of combined hepatocellular and cholangiocarcinoma with hepatocellular carcinoma and intrahepatic cholangiocarcinoma. Surg Today. 2006;36(10): 892-7.

2. Lee JH, Chung GE, Yu SJ, Hwang SY, Kim JS, Kim HY, Yoon JH, Lee HS, Yi NJ, Suh KS, et al. Long-term prognosis of combined hepatocellular and cholangiocarcinoma after curative resection comparison with hepatocellular carcinoma and cholangiocarcinoma. J Clin Gastroenterol. 2011;45(1):69-75.

3. Allen RA, Lisa JR. Combined liver cell and bile duct carcinoma. Am J Pathol. 1949;25(4):647-55.

4. Zhang F, Chen XP, Zhang W, Dong HH, Xiang S, Zhang WG, Zhang BX. Combined hepatocellular cholangiocarcinoma originating from hepatic progenitor cells: immunohistochemical and double-fluorescence immunostaining evidence. Histopathology. 2008;52(2):224-32.

5. Itoyama M, Hata M, Yamanegi K, Yamada N, Ohyama H, Hirano H,Terada N, Nakasho K. Expression of both hepatocellular carcinoma and cholangiocarcinoma phenotypes in hepatocellular carcinoma and cholangiocarcinoma components in combined hepatocellular and cholangiocarcinoma. Med Mol Morphol. 2012:45(1):7-13.
6. Fujii H, Zhu XG, Matsumoto T, Inagaki M, Tokusashi Y, Miyokawa N, Fukusato T, Uekusa T, Takagaki T, Kadowaki N et al. Genetic classification of combined hepatocellular-cholangiocarcinoma. Hum Pathol. 2000;31(9):1011-7.

7. Kim H, Park C, Han KH, Choi J, Kim YB, Kim JK, Park YN.: Primary liver carcinoma of intermediate (hepatocyte-cholangiocyte) phenotype. J Hepatol. 2004:40(2):298-304.

8. Tanaka S, Yamamoto T, Tanaka H, Kodai S, Ogawa M, Ichikawa T Hai S, Sakabe K, Uenishi T, Shuto T, et al. Potentiality of combined hepatocellular and intrahepatic cholangiocellular carcinoma originating from a hepatic precursor cell: Immunohistochemical evidence. Hepatol Res. 2005;32(1):52-7.

9. Shetty AS, Fowler KJ, Brunt EM, Agarwal S, Narra VR, Menias CO. Combined hepatocellular-cholangiocarcinoma: what the radiologist needs to know about biphenotypic liver carcinoma. Abdom Imaging. 2014;39(2):310-22.

10. Lee CH, Hsieh SY, Chang CJ, Lin YJ. Comparison of clinical characteristics of combined hepatocellular-cholangiocarcinoma and other primary liver cancers. J Gastroenterol Hepatol. 2013;28(1):122-7.

11. de Campos RO, Semelka RC, Azevedo RM, Ramalho M, Heredia V, Armao DM, Woosley JT. Combined hepatocellular carcinoma-cholangiocarcinoma: report of MR appearance in eleven patients. J Magn Reson Imaging. 2012; 36(5):1139-47.

12. Hwang J, Kim YK, Park MJ, Lee MH, Kim SH, Lee WJ, Rhim HC. Differentiating combined hepatocellular and cholangiocarcinoma from mass-forming intrahepatic cholangiocarcinoma using gadoxetic acid-enhanced MRI. J Magn Reson Imaging. 2012;36(4):881-9.

13. Ebied O, Federle MP, Blachar A, Brancatelli G, Grazioli L, Cazals-Hatem D, Dondero F, Vilgrain V. Hepatocellular-cholangiocarcinoma: helical computed tomography findings in 30 patients. J Comput Assist Tomogr. 2003;27(2):117-24.

14. Nishie A, Yoshimitsu K, Asayama Y, Irie H, Aibe H, Tajima T, Shinozaki K, Nakayama T, Kakihara D, Shimada M et al. Detection of combined hepatocellular and cholangiocarcinomas on enhanced CT: comparison with histologic findings. AJR Am J Roentgenol. 2005;184(4):1157-62.

15. Fowler KI, Sheybani A, Parker RR, Doherty S, M Brunt E, Chapman WC, Menias CO. Combined hepatocellular and cholangiocarcinoma (biphenotypic) tumors: imaging features and diagnostic accuracy of contrast-enhanced CT and MRI. AJR Am J Roentgenol. 2013;201 (2):332-9.

16. Kassahun WT, Hauss J. Management of combined hepatocellular and cholangiocarcinoma. Int J Clin Pract. 2008;62(8):1271-8.

17. Tang D, Nagano H, Nakamura M, Wada H, Marubashi S, Miyamoto A Takeda Y, Umeshita K, Dono K, Monden M. Clinical and pathological features of Allen's type $C$ classification of resected combined hepatocellular and cholangiocarcinoma: a comparative study with hepatocellular carcinoma and cholangiocellular carcinoma. J Gastrointest Surg. 2006; 10(7):987-98.

18. Jarnagin WR, Weber S, Tickoo SK, Koea JB, Obiekwe S, Fong Y, DeMatteo RP, Blumgart LH, Klimstra D. Combined hepatocellular and cholangiocarcinoma: demographic, clinical, and prognostic factors. Cancer Am Cancer Soc. 2002; 94(7):2040-6.

19. Gibson JB. Histological typing of tumors of the liver, biliary tract, and pancreas. Geneva: World Health Organization; 1987.

20. Theise N, Nakashima O, Park YN, Nakanuma Y. Combined hepatocellularcholangiocarcinoma: WHO classification of tumours of the digestive system. Lyon, France: IARC; 2010. p. 225-7.

21. Claudon M, Cosgrove D, Albrecht T, Bolondi L, Bosio M, Calliada F, Correas JM, Darge K, Dietrich C, D'Onofrio M et al. Guidelines and good clinical practice recommendations for contrast enhanced ultrasound (CEUS) - update 2008. Ultraschall Med. 2008;29(1):28-44.

22. Claudon M, Dietrich CF, Choi Bl, Cosgrove DO, Kudo M, Nolsoe CP, Piscaglia F, Wilson SR, Barr RG, Chammas MC et al. Guidelines and good clinical practice recommendations for contrast enhanced ultrasound (CEUS) in the liver-update 2012: a WFUMB-EFSUMB initiative in cooperation with representatives of AFSUMB, AIUM, ASUM, FLAUS and ICUS. Ultraschall Med. 2013;34(1):11-29.

23. Boozari B, Soudah B, Rifai K, Schneidewind S, Vogel A, Hecker H, Hahn A, Schlue J, Dietrich CF, Bahr MJ et al. Grading of hypervascular hepatocellular carcinoma using late phase of contrast enhanced sonography - a prospective study. Dig Liver Dis. 2011:43(6):484-90.

24. Li R, Yuan MX, Ma KS, Li XW, Tang CL, Zhang XH, Guo DY, Yan XC. Detailed analysis of temporal features on contrast enhanced ultrasound may help differentiate intrahepatic cholangiocarcinoma from hepatocellular carcinoma in cirrhosis. Plos One. 2014;9(5):e98612. 
25. Kong WT, Wang WP, Huang BJ, Ding H, Mao F. Value of wash-in and washout time in the diagnosis between hepatocellular carcinoma and other hepatic nodules with similar vascular pattern on contrast-enhanced ultrasound. J Gastroenterol Hepatol. 2014;29(3):576-80.

26. Rimola J, Forner A, Reig M, Vilana R, de Lope CR, Ayuso C, Bruix J. Cholangiocarcinoma in cirrhosis: absence of contrast washout in delayed phases by magnetic resonance imaging avoids misdiagnosis of hepatocellular carcinoma. Hepatology. 2009;50(3):791-8.

27. lavarone M, Piscaglia F, Vavassori S, Galassi M, Sangiovanni A, Venerandi L, Forzenigo LV, Golfieri R, Bolondi L, Colombo M. Contrast enhanced CT-scan to diagnose intrahepatic cholangiocarcinoma in patients with cirrhosis. J Hepatol. 2013;58(6):1188-93.

28. Bruix J, Sherman M. Management of hepatocellular carcinoma: an update. Hepatology. 2011;53(3):1020-2.

29. Yano Y, Yamamoto J, Kosuge T, Sakamoto Y, Yamasaki S, Shimada K, Ojima H, Sakamoto M Takayama T, Makuuchi M. Combined hepatocellular and cholangiocarcinoma: a clinicopathologic study of 26 resected cases. Jpn J Clin Oncol. 2003;33(6):283-7.

30. Ng IO, Shek TW, Nicholls J, Ma LT. Combined hepatocellular-cholangiocarcinoma: a clinicopathological study. J Gastroenterol Hepatol. 1998;13(1):34-40.

31. Taguchi J, Nakashima O, Tanaka M, Hisaka T, Takazawa T, Kojiro M. A clinicopathological study on combined hepatocellular and cholangiocarcinoma. J Gastroenterol Hepatol. 1996;11(8):758-64.

32. Liu CL, Fan ST, Lo CM, Ng IO, Lam CM, Poon RT, Wong J. Hepatic resection for combined hepatocellular and cholangiocarcinoma. Arch Surg. 2003;138(1):86-90.

33. Lee CC, Wu CY, Chen JT, Chen GH. Comparing combined hepatocellularcholangiocarcinoma and cholangiocarcinoma: a clinicopathological study. Hepatogastroenterology. 2002;49(48):1487-90.

34. Yin X, Zhang BH, Qiu SJ, Ren ZG, Zhou J, Chen XH, Zhou Y, Fan J. Combined hepatocellular carcinoma and cholangiocarcinoma: clinical features, treatment modalities, and prognosis. Ann Surg Oncol. 2012;19(9):2869-76.

35. Kim SA, Lee JM, Lee KB, Kim SH, Yoon SH, Han JK, Choi BI. Intrahepatic mass-forming cholangiocarcinomas: enhancement patterns at multiphasic $C T$, with special emphasis on arterial enhancement pattern-correlation with clinicopathologic findings. Radiology. 2011;260(1):148-57.

36. Yu XH, Xu LB, Zeng H, Zhang R, Wang J, Liu C. Clinicopathological analysis of 14 patients with combined hepatocellular carcinoma and cholangiocarcinoma. Hepatobiliary Pancreat Dis Int. 2011;10(6):620-5.

\section{Submit your next manuscript to BioMed Central and we will help you at every step:}

- We accept pre-submission inquiries

- Our selector tool helps you to find the most relevant journal

- We provide round the clock customer support

- Convenient online submission

- Thorough peer review

- Inclusion in PubMed and all major indexing services

- Maximum visibility for your research

Submit your manuscript at www.biomedcentral.com/submit 